\title{
Interaction between Mental Rotation and Manual Rotation with and without Motor Planning
}

\author{
Shinkichi Nishihara', Fumihito Imai' ${ }^{2}$, Akiko Fujiki², Yoshimasa Majima ${ }^{3}$ \\ ${ }^{1}$ Hokkaido University Center for Environmental and Health Sciences, Sapporo, Japan \\ ${ }^{2}$ Department of Psychology, Graduate School of Letters Hokkaido University, Sapporo, Japan \\ ${ }^{3}$ Hokusei Gakuen University, Sapporo, Japan \\ Email: nishihara.psych@gmail.com
}

Received 2 June 2015; accepted 6 July 2015; published 13 July 2015

Copyright (C) 2015 by authors and Scientific Research Publishing Inc.

This work is licensed under the Creative Commons Attribution International License (CC BY). http://creativecommons.org/licenses/by/4.0/

c) (i) Open Access

\begin{abstract}
Two experiments involving mental rotation with two types of manual rotation were run. In Exp 1, participants had to perform the mental rotation task with active hand movement using a rotating device that did not move automatically. In Exp 2, participants had to perform the mental rotation with passive hand movement using an automatically rotating device. The interaction effect between mental and manual rotation was observed only in the former case. These opposing results indicate that it is motor planning associated only with active movement that plays an important role in the interaction between mental and manual rotation and not the proprioceptive/kinesthetic feedback caused by the manual rotation itself.
\end{abstract}

\section{Keywords}

Mental Rotation, Manual Rotation, Motor Planning, Kinesthetic Feedback, Active Hand Movement, Passive Hand Movement

\section{Introduction}

Mental imagery is defined as quasi-perceptual experience such as "seeing" in the absence of the appropriate immediate sensory input (e.g., Kosslyn, Behrmann, \& Jeannerod, 1995). It has been revealed that some kinds of mental imagery share mechanisms with action (e.g., Pearson, 2001; Pearson, Logie, \& Gilhooly, 1999). Mental rotation of visual stimulus is a good example of such cognitive activity. It occurs when someone compares two visual stimuli (e.g., 3D cube array objects; Shepard \& Metzler, 1971) whose orientations differ from each other 
(e.g., the comparison stimulus is made by having rotated the standard stimulus some degrees). In such a case, she/he mentally rotates one of the stimuli to the same orientation of its counterpart as if the rotation follows the physical law. So, as with physical rotation, the reaction time that she/he needs to judge whether two stimuli are identical or not (i.e., comparison stimulus is a mirror image of standard stimulus) increases in proportion to the angular difference between the two stimuli (e.g., Shepard \& Metzler, 1971).

Some previous findings suggested that such mental rotation shared some mechanisms with action, especially motor planning. For example, Wexler, Kosslyn \& Berthoz (1998) and Wohlschläger \& Wohlschläger (1998) showed that concurrent manual rotation relatively facilitated mental rotation in the same direction (e.g., clockwise or counterclockwise rotation) compared with the case of the opposite direction. In both the studies, this interaction was interpreted as indicating that mental and manual rotations shared some motor process, and it was assumed that the interaction would occur at a relatively high level of motor processing like motor planning. In a further study, Wohlschläger (2001) directly investigated the relationship between motor planning and mental rotation. He asked the participant to rotate the part of a device (knob) in the preliminarily indicated direction (i.e., clockwise or counterclockwise), but its execution was postponed until having completed the mental rotation task. That is, unlike previous studies (Wexler, Kosslyn, \& Berthoz, 1998; Wohlschläger \& Wohlschläger, 1998), the manual rotation was not executed during a mental rotation task. Thus, if there were an interaction between mental and manual rotations, it meant that only motor planning of a hand movement was able to affect the performance on the mental rotation task. The results indicated that the preparation for the manual rotation (without execution) produced a similar interaction pattern of reaction time as that observed in the previous studies. As described above this result was interpreted to mean that the planning of rotational hand movements played an important role in mental rotation.

Although Wohlschläger (2001) has demonstrated the importance of motor planning in mental rotation using well-established experimental methods, the role of hand movement itself without motor planning (i.e., a kinesthetic feedback) remains unclear. In his paper, he pointed out that the interaction between manual and mental rotations could be based on a cross-modal interference between visuo-spatial imagery and the kinesthetic feedback elicited by the movements. Kinesthetic feedback should be distinguished from a motor plan itself because a bare motor plan is either a dynamic plan (a temporal sequence of motor commands or muscle tensions) or a kinematic plan (a plan for limb movement specified in terms of joint angles) that is different from proprioceptive/ kinesthetic feelings (Grush, 2004). Therefore, Wohlschläger's experiment where no hand movement was executed during mental rotation did not test any effect of a movement itself (i.e., kinesthetic feedback). In addition, he reported that the interaction observed in his study was weaker than that in the previous study where motor planning was accompanied by the execution (Wohlschläger \& Wohlschläger, 1998). This difference might have been due to the absence of a motor execution in the experiment of Wohlschläger (2001). In other words, a movement itself might have some additional effect on mental rotation performance. Thus, it is important to clarify whether the actual hand movement without motor plan can be related to the processing of the mental rotation.

From this point of view, the present study investigates whether the manual rotation with (experiment 1 ) and without a motor plan (experiment 2) affects the performance of the mental rotation task. If both types of manual rotations affect the performance on a mental rotation task, it will indicate that not only the motor plan but also proprioceptive/kinesthetic feedback elicited by the movement plays some additional role in the processing of the mental rotation. On the other hand, if the rotational hand movement without a motor plan has no effect on the performance, it must strongly indicate that motor planning is the nature of the mental rotation processing.

It is methodologically important to control active and passive aspects of the task (e.g., Quinn \& Ralston, 1986). For this purpose, we adopted active and passive hand movements by inventing a rotational device (see apparatus in Method of Exp 1 and 2). In Experiment 1, participants performed the mental rotation task while rotating the device voluntarily (i.e., active hand movement). On the other hand, in Experiment 2, participants performed a similar dual task but the second task was to keep hold of an automatically rotated device (i.e., passive hand movement). Thus, active movement involved both motor plan and proprioceptive/kinesthetic feedback, while passive movement involved only proprioceptive/ kinesthetic feedback.

Finally, it is foreseeable that controlling the involvement of passive and active aspects in the mental rotation task might raise an extraneous variable, like differences of mental efforts. If so, it should be important to prepare a measure of detecting the effect of "mental effort" in the performance of 2 tasks. So, the present study adopted the additional experimental conditions. In both Experiment 1 and 2, to demonstrate whether the mental effort to 
project their hand movement on the display was effective or not, participants were divided into two experimental groups: mental and non-mental effort groups. In the former group, participants must make effort to project their hand movement on the display. In particular, participants received additional instruction to enhance the possible interaction between visuo-spatial imagery (i.e., mental rotation) and the kinesthetic feedback caused by movement itself. Participants were required to imagine a rotation of a presented directional stimulus (i.e., an arrow) synchronized with their hand movement (regardless of manual or automatic one). This manipulation was expected to enhance assumed interaction between visual-spatial imagery and kinesthetic feedback. In the latter group, they did not need to make such efforts. It would be instructive to detect an effect of purely kinesthetic feedback to emphasize the difference of involvement of mental effort.

\section{Experiment 1}

Experiment 1 was designed to demonstrate the effect of a manual rotation (i.e, a hand movement with a motor plan) on the mental rotation task performance. According to Wohlschläger \& Wohlschläger (1998), it was predicted that the manual rotation affected the performance on the mental rotation task. As described in introduction, we wished to distinguish an active aspect from a passive one in the manual rotation. We adopted the levertype device instead of the knob-type one such as Wohlschläger \& Wohlschläger (1998) used because the knob rotation constrained a participant to re-grasp the device periodically and it, itself, needed some motor plan. On the other hand, as shown in Exp 2, our handmade device enabled a participant to accomplish an automatic hand movement without such a motor plan only by grasping the lever in Experiment 2.

\subsection{Method}

\subsubsection{Participants}

Twenty-four student volunteers at Hokkaido University participated in this experiment (12 females and 12 males). All were right-handed and unaware of the purpose of the experiment. They had normal or corrected-tonormal vision for each eye and reported no vision defects.

\subsubsection{Apparatus}

All visual stimuli (mental rotation stimuli and instructional icons) were presented on a 15-inch display with full screen mode. A personal computer (iMac G4 PowerPC 7450) and experimental software (Super Lab 1.77) were used to control presentation of the stimuli. The display was on the desk approximately $80 \mathrm{~cm}$ away from a participant. The viewing angles were about $16^{\circ}$ in the vertical and $21^{\circ}$ in the horizontal.

The rotating device for performing the manual rotation was composed of a wooden stick, an acrylic pipe and a metal wheel. There was a metal fixture at the center of the acrylic plate and participants could rotate the lever around the fixture point. In order to conduct flexible and smooth manual rotation (without re-grasping the lever), the wooden lever was inserted into the acrylic pipe instead of being strapped into it (Figure 1).

A cover box made of corrugated paper prevented the participants from viewing their manual rotational movement. In addition, a microphone attached to each participant's throat received his or her oral response, and a keyboard was used by the experimenter controlling the iMac.

\subsubsection{Visual Stimuli}

Mental rotation stimuli were created by Adobe Photoshop 5.0. Those were normal or mirror-reversed images of the letter $F, G, J, L, P, R$ rotated in steps of $60^{\circ}$. Thus in total 72 visual stimuli were used in Experiment 1 . All of mental rotation stimuli were presented at approximately $4^{\circ}$ visual angle. In addition, three types of icons were used for instructing the direction of the manual rotation (instructional icons); clockwise, counterclockwise (i.e., arrows curved to each direction), and no rotation (i.e., a black dot).

\subsubsection{Task}

Using these apparatus and visual stimuli, the mental rotation and the manual rotation tasks were conducted simultaneously as a dual task experiment.

In the experimental task, there were 216 trials that were divided into 6 blocks. Normal or mirror-reversed images of a letter presented in 6 angles $\left(0^{\circ}, 60^{\circ}, 120^{\circ}, 180^{\circ}, 240^{\circ}, 300^{\circ}\right)$ were used in each block. In combination with these mental rotation stimuli, three types of hand movement conditions (clockwise rotation, counterclock- 


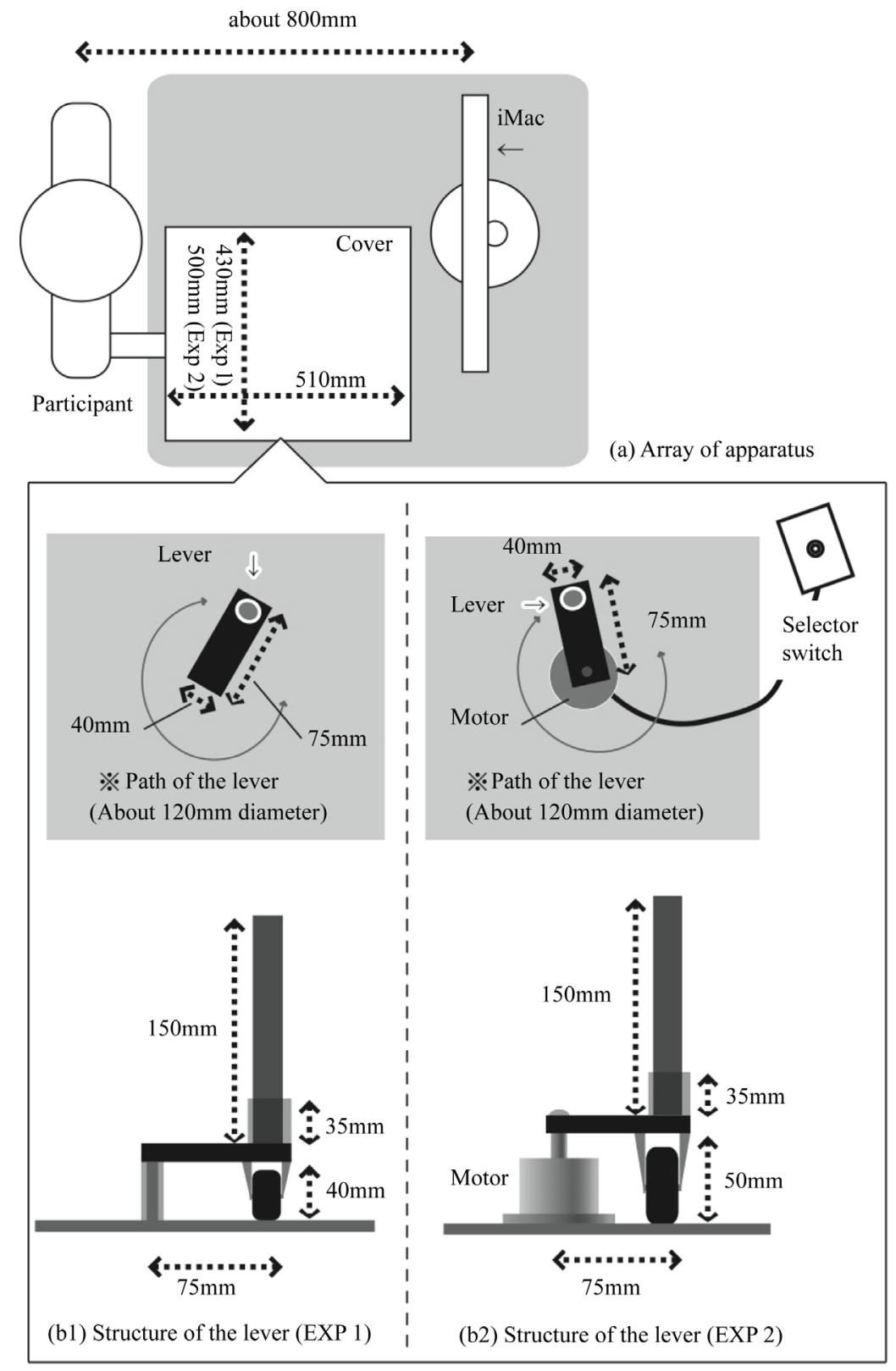

Figure 1. Apparatus (Experiment 1 \& 2).

wise rotation, no rotation) were performed. The order of the stimulus presentation was randomized in each block. The randomized block design was used to decide the block order in the experiment.

\subsubsection{Procedure}

Each Participant was seated in a dimly lit room. A desk was in front of him or her. After an instruction, a practice session was conducted. This session consisted of two phases. The one was a hand movement-learning phase where the appropriate rotational speed of the manual rotation was learned. The other was practice phase where the whole trial flow was practiced.

In the hand movement-learning phase, the participant grasped the lever stick and performed one rotation per second. The rotational speed was controlled by a metronome. In the practice phase, the participant engaged in the mental rotation task with manual rotation. Practice consisted of 18 trials and normal or mirror-reversed images of digit " 2 " were used as a mental rotation stimulus. 
After the practice session, the experimental session started (Figure 2). The participant was asked to hold the lever using his/her right hand while looking at the fixation cross at the center of the display. Each trial was initiated after confirming the participant was fully ready. At the beginning of the trial, one of the three instructional icons was presented for 1000 msec. The meanings of each instructional icon were as follows: one was a clockwise arrow that indicated a clockwise direction (cw); another was a counterclockwise arrow that indicated a counterclockwise direction (ccw); the other was a black dot that meant no hand movement (none). As soon as the participants saw an instructional icon, they had to rotate the lever in the indicated direction or keeping the lever stay on the start point. The lever rotation was continued up to the end of the trial.

The mental rotation task was started 4 seconds after the instructional icon disappeared. In the mental rotation task, the participant had to continue to rotate their hand and judge whether the presented letter was normal or mirror-reversed as fast and accurately as possible. When it was a normal image, the participant had to say, "Yes" otherwise "No". The microphone attached to the participant's throat measured response time. At the same time, the experimenter recorded the participant's answer.

The participants were divided into the 2 groups (6 females and 6 males for each mental effort group). Participants in mental effort group were instructed to imagine a rotation of a presented instructional icon (i.e., an arrow) synchronized with their hand movement (regardless of manual or automatic one). In the non-mental effort group, participants received no such an instruction. These groups were set in order to clarify whether deliberate effort to synchronize the hand movement onto the visual display affects the performance on the mental rotation task.

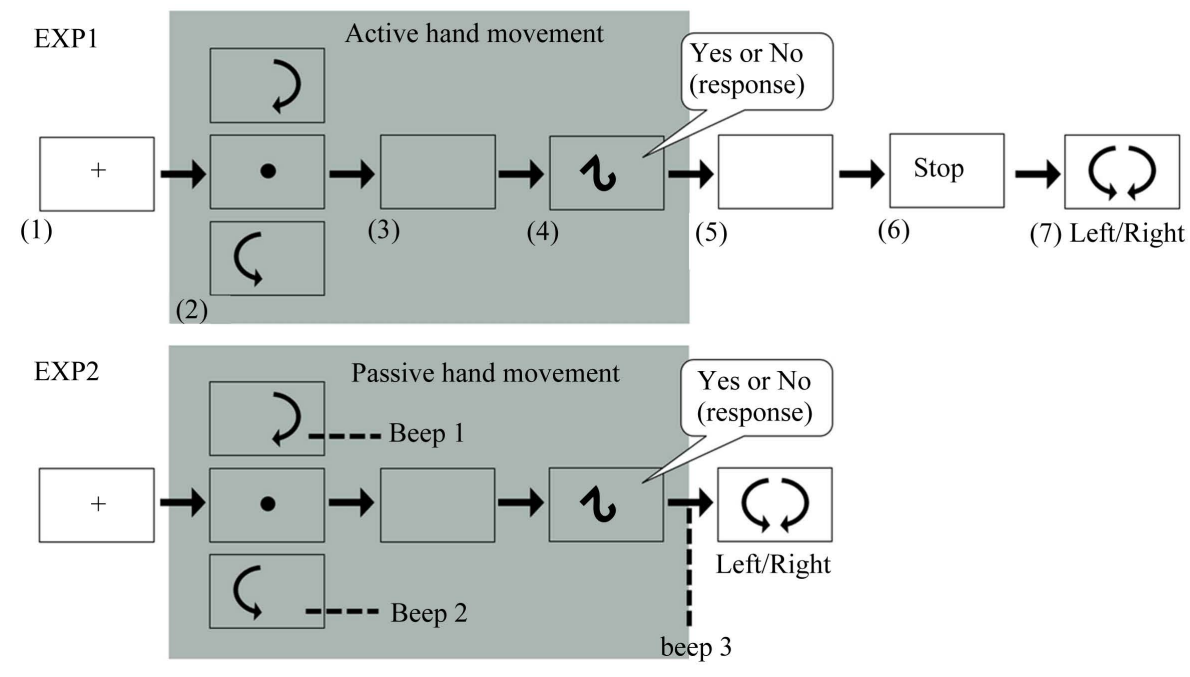

Figure 2. Procedure of Experiment 1 \& 2. (1) The fixation mark appeared on the center of the display. If the participant has been ready to start, the experimenter has started up the trial. (2) Either a curved arrow or a dot appeared. In the case of a dot, no lever movement was done. In the case of an arrow; a) in EXP1, the participant moved the lever toward a direction indicated by the arrow (about 1 rotation/s). (b) In EXP2, the lever that the participant holds started automatic rotation with the beep sound (Beep 1 for clockwise rotation, Beep 2 for counter-clockwise rotation) (about 1 rotation/s). In both the experiments, Imagery group participants were instructed to imagine the rotation of the arrow during manual/automatic rotation. Both an arrow and a dot disappeared soon. (3) The participant kept fixation with a lever rotation or without it. (4) After a certain period of time, a letter appeared on the display. The participant answered "No" if the letter was mirror image, and "Yes" if it was correct image with active (EXP1) or passive (EXP2) hand movement. He/she was instructed to answer as soon and accurate as possible. In EXP2, after the answer, the automatic lever rotation was stopped (accompanied with the sound "Beep 3".) (5) The letter disappeared after participant's answer. Only in EXP1, the participant kept fixation with or without an active hand movement. (6) Only in the manual rotation condition of EXP1, the message that asked participant to stop a lever rotation appeared, and he/she stopped it. (7) After that, two curved arrows appeared. The participant answered the direction of his/her letter mental rotation. Clockwise direction was "right", counterclockwise was "left", no intentional mental rotation (and forgot the mental rotation direction) was "pass". His/her answer triggered next trial with appearing new fixation mark. 


\subsubsection{Design}

A 3 (manual rotation direction; counterclockwise or clockwise or no manual rotation) $\times 6$ (angles of mental rotation stimulus, in steps of $60^{\circ}$ ) $\times 2$ (group: mental effort or non-mental effort group) factorial design was used. Manual rotation direction and angles were within-participants factors, and group was a between-participant factor.

\subsection{Results and Discussion}

The median response times of correct answers were analyzed using a three-way ANOVA. An alpha level .05 was adopted. The ANOVA revealed a main effect of angles $(F(5,110)=6.26, p<0.001)$ and an interaction effect between angles and manual rotation directions $(F(10,220)=2.54, p<0.01)$ (Figure 3). The multiple comparison with the Ryan's method of the interaction effect revealed that both counterclockwise $(t(264)=1.97, p<$ $0.05)$ and clockwise $(t(264)=2.81, p<0.001)$ manual rotation facilitated the mental rotation performance at angle $180^{\circ}$ compared with the control condition. In addition, only counterclockwise manual rotation impaired the performance at angle $240^{\circ}$ compared to the clockwise condition $(t(264)=3.05, p<0.01)$ and control condition $(t(264)=3.29, p<0.01)$.

According to the previous studies (e.g., Wexler, Kosslyn, \& Berthoz, 1998; Wohlschläger \& Wohlschläger, 1998), manual rotation had an influence on the mental rotation performance. The results of Experiment 1 were consistent with these findings. That is manual hand movement gave positive (i.e., MR stimuli were presented at angle $180^{\circ}$ ) and negative effects (MR stimuli were presented at angle $240^{\circ}$ ) respectively. It must be appropriate to interpret that the concurrent manual hand movement gave positive and negative effects respectively.

In visual stimulus presentation angle $180^{\circ}$, there was neither advantage nor disadvantage regardless of participant rotating the visual stimulus in any direction as long as the directions of mental and manual rotations were congruent. So, the facilitative effects were simply observed in both directions in presentation angle $180^{\circ}$. On the other hand, in visual stimulus presentation angle $240^{\circ}$, it was completely natural to think that there is a strong advantage for the participants to rotate a visual stimulus to the clockwise direction, because participants only have to rotate it 120 degree toward the clockwise direction that was found by Shepard \& Metzler (1971). Therefore, only counterclockwise manual rotation had negative influence on the processing of mental rotation. On the other hand, there were no effects at the other angles. These results might have been caused by the task difficulty. It was thought that these angles were too easy to detect the effect of manual hand movement.

In addition, there were no effects related to the mental effort groups in Experiment 1 . This result means that

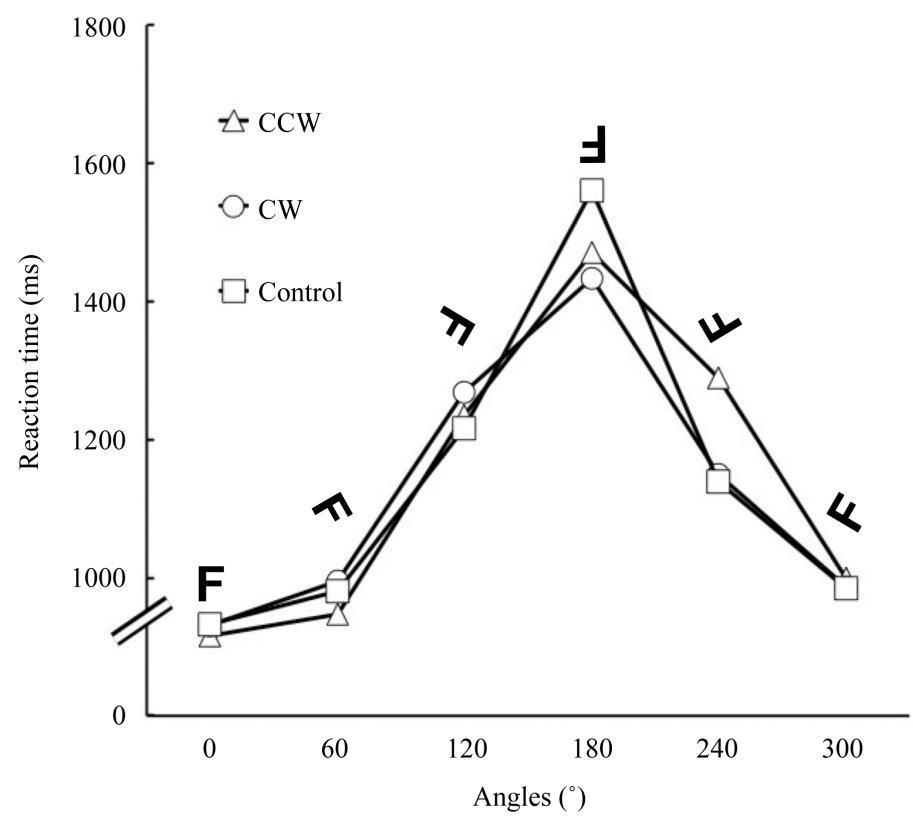

Figure 3. Relationship between angles and directions of manual rotation. 
the mental effort to project manual hand movement on the visual display is not effective at least in this experimental situation.

The type of manual rotation adopted in Experiment 1 was composed of motor planning and hand movement itself (i.e., kinesthetic feedback). Thus it is possible that both motor planning and kinesthetic feedback would affect the results observed in this experiment. As described in introduction, in the present study it was intended to investigate whether hand movement itself (i.e., kinesthetic feedback) would have some influence on the mental rotation performance. Therefore, in Experiment 2, we tested whether passive hand movement without the motor planning influence on the processing of mental rotation.

\section{Experiment 2}

\subsection{Method}

\subsubsection{Participants}

Twenty-four student volunteers at Hokkaido University (who were not participants in Exp 1) participated in this experiment (12 females and 12 males). All were right-handed and unaware of the purpose of the experiment. All had normal or corrected-to-normal vision for each eye and reported no vision defects.

\subsubsection{Apparatus and Visual Stimuli}

All apparatus other than the rotating device were the same as Experiment 1. The rotating device consisted of a wooden stick (lever), an acrylic pipe, a metal wheel, stepping motor, shield wire, and switching box. The stepping motor was set at the center of the acrylic plate and the rotation of the device was driven by the motor. The switching box was attached to the stepping motor with shield wire and the experimenter controlled the rotated direction (ccw/cw/none) using the switching box. While a participant held the lever, his/her hand was passively rotated in a specific direction (one rotation per second). As in Experiment 1, in order to achieve a flexible and smooth manual rotation, the wooden lever was just inserted into acrylic pipe instead of being strapped into it (Figure 1). All visual stimuli (mental rotation stimuli and instruction icons) were the same as Experiment 1.

\subsubsection{Task}

Using these apparatus and visual stimuli, the mental rotation task and the passive manual rotation task were conducted simultaneously as a dual task experiment.

As the experimental task, there were 216 trials divided into 6 blocks. Normal or mirror-reversed images of a letter presented in 6 angles $\left(0^{\circ}, 60^{\circ}, 120^{\circ}, 180^{\circ}, 240^{\circ}, 300^{\circ}\right)$ were used as mental rotation stimuli in each block. In combination with these mental rotation stimuli, three types of passive hand movement (clockwise rotation, counterclockwise rotation, no rotation) were performed. The order of the stimulus presentation was randomized in each block. The randomized block design was used to decide the block order in this experiment.

\subsubsection{Procedure}

The participant was seated in a dimly lit room. A desk was in front of him/her. After instructions, the practice session was conducted. This session consisted of two phases. The one was the hand movement practice phase and the other was the practice phase.

In the hand movement practice phase, passive manual rotation was learned. Because of motor driven rotation, passive hand movement was accomplished while participants only kept holding the lever. In the practice phase, the whole trial flow was learned. The participant engaged in the mental rotation task with the passive manual rotation. This practice was composed of 18 trials and normal or mirror-reversed images of the digit "2" were used as a mental rotation stimulus.

After the practice session, the experimental session started (Figure 2). The participants were asked to keep holding the lever using their right hand while looking at the fixation cross at the center of the screen. Each trial was initiated after confirming the participant was fully ready. At the beginning of trial, one of the three instructional icons indicating the passive manual rotation direction was presented for 1000 msec. The meaning of each instructional icon was same as Experiment 1 . As soon as an instructional icon was presented, the device started to rotate automatically in the indicated direction (ccw/cw/none). The passive rotation was continued up to the end of the trial.

The mental rotation task was started 4 seconds after the instructional icon disappeared. While the participant 
was performing the mental rotation task, the passive hand rotation was maintained. In the mental rotation task, participants were asked to judge whether the presented letter was normal or mirror-reversed as fast and accurate as possible. When it was a normal image, participant had to say, "Yes" otherwise "No". The microphone attached to the participant's throat measured the response time. At the same time, the experimenter recorded the participant's answer.

As in Experiment 1 the participants were divided into the 2 groups (6 females and 6 males in mental effort group and 6 female and 6 male in non-mental effort group). Participants in mental effort group were instructed to imagine a rotation of a presented stimulus (i.e., an arrow) synchronized with their hand movement (regardless of manual or automatic one). In the non-mental effort group, participants received no such instruction. These groups were set in order to clarify whether deliberate effort to combine the hand movement and visual processing affect the mental rotation performance.

\subsubsection{Design}

The experiment was performed with two within-subjects (angle and manual rotation direction) and one betweensubject (group; mental effort or non-mental effort group) factorial design that was the same as Experiment 1.

\subsection{Results and Discussion}

As was the case in Experiment 1, the task performance was defined as the median response time of correct answer in each condition. The data were analyzed using a three-way ANOVA. An alpha level .05 was adopted. This ANOVA revealed only a typical main effect of angles $(F(5,110)=2.19, p<0.001)$ and there were no other main or interaction effects. This result indicates that the differences of presentation angle had influenced on the response time like Shepard and Metzler (1971), however, manual rotation had no influence on it.

The type of manual rotation adopted by Experiment 2 would not be related to the motor planning. In other words, this passive hand movement was composed of kinesthetic feedback only. The results of this experiment indicate that kinesthetic feedback itself does not have any influence on the mental rotation performance.

In addition, there were no effects related to the mental effort groups in Experiment 2. This result means that the mental effort to project manual hand movement on the visual display is not effective at least in this experimental situation.

\section{General Discussion}

The present study investigated what part of the motor system played an important role in mental rotation, with the experiment specifically focused on motor planning and kinesthetic feedback.

The results of Experiment 1 and 2 indicated that the planning of hand movement systematically affected the mental rotation performance (i.e., both positive and negative effects), however, kinesthetic feedback of hand movement did not play any important role in mental rotation.

Previous studies indicated the importance of motor planning in the manual rotation (e.g., Wexler, Kosslyn, \& Berthoz, 1998; Wohlschläger, 2001; Wohlschläger \& Wohlschläger, 1998). However, they did not verify the possibility that kinesthetic feedback had some important effects on the processing of mental rotation. To investigate the pure effect of kinesthetic feedback (without an effect of motor planning), it was important for the present study to control the involvement of motor planning in manual rotation. This is why we introduced the handmade device that accomplishes active and passive manual rotations into the study. In Experiment 1, we investigated whether manual rotation including both motor planning and kinesthetic feedback affected the mental rotation performance. The result indicated that this type of manual rotation had both the positive and negative effects on the processing of mental rotation. Picking up details in Experiment 1, however, the interaction effects were observed only in the $180^{\circ}$ (i.e., positive effect) and $240^{\circ}$ angles (i.e., negative effect). Therefore, the effect of manual rotation seemed to have been weaker than that in previous studies (Wohlschläger, 2001; Wohlschläger \& Wohlschläger, 1998). Compared with these studies, the following factors may have decreased the effect of manual rotation: First, because the present study adopted letter images as mental rotation stimuli, it might have made the task easier. As proof of this, positive and negative effects of manual rotation were observed as interaction effects only in $180^{\circ}$ and $240^{\circ}$ angles that were relatively difficult compared with the other angles. Second, according to Wohlschläger (2001) and Wohlschläger \& Wohlschläger (1998), the difference axis between manual movement (on the desk) and mental rotation of visual stimulus (on the display) would make the interaction 
effect weaker. In fact, participants in the present study performed manual rotation on the desk plane while performing mental rotation on the display plane that was perpendicular to the former one. Thus it is possible that the difference of axis between mental rotation and manual rotation in Experiment 1 reduced the interaction effect. Nevertheless, the interaction effect observed in Experiment 1 showed a quite systematic pattern; at the $180^{\circ}$ angle, both the cw and ccw manual rotation provided a strong positive effect, while counterclockwise rotation gave a negative effect in $240^{\circ}$ angle. This is the reason we regard this result as quite important. In modern daily life with a personal computer, we can naturally synchronize mouse movement (i.e., on a desk) to the cursor movement (i.e., on the display) beyond the difference of two axes. Taking into account this example of our daily life, the axis difference would not weaken the interaction effect between motor system and visual system. If an experimenter could construct the experimental situation with high ecological validity like controlling mouse cursor by moving a mouse, the interaction should emerge even if the axes are different. So we developed the rotating device with careful attention that the device could move more smoothly like a mouse, because smoothness could make the projection of motor sensory image onto the display easy. We believed that the smooth hand movement using our rotating device moderated the effects of the different rotational axes. Indeed, a systematic interaction effect was observed in Experiment 1. This result indicates that the axis difference would not be an important factor of the interaction effect between manual and mental rotations. Furthermore, the effect of axis in Wohlschläger \& Wohlschläger (1998) would be nothing more than being caused by the rotational quality of the knob device. Considering our daily life, it is quite rare for us to manipulate something using knob types of rotating device. So, the interaction effects between knob rotation and mental rotation would be observed only in the same axis. The mental effort of projecting manual movement onto a visual display, however, had no significant effect in this study. According to such results, the mental effort would not be an important factor of the interaction between manual and mental rotations in such a situation. In any case, further investigation about this problem is required.

On the other hand, manual rotation without motor planning gave no interaction effect in Experiment 2. This result suggests that manual rotation only with kinesthetic feedback does not have any effects on the processing of mental rotation. This finding should strongly support the notion of Wohlschläger (2001), and indicates that kinesthetic feedback that plays an important role in motor control is not important for the mental manipulation of visual stimulus that occurs in mental rotation.

\section{Conclusion}

In conclusion, the present study showed the different effects of active and passive hand movements on the visuo-spatial task. Mental rotation is thought to be a typical manipulation task of visual imagery and visual short-term memory. In the Logie's (1995) model of visuo-spatial working memory, the inner scribe is assumed to be responsible for the manipulation of visual imagery. In addition, the inner scribe is also related to motor planning and according to the result of Quinn \& Ralston (1986), only active movement would affect the function of inner scribe. If the relationship between mental and manual rotations can be explained in the framework of VSWM, it is instructive to remember the situation in which we manipulate our mental imagery. When we control our visual imagery, the actual hand movement should not be necessary. The kinesthetic feedback itself is not the necessary condition for the interaction between motor system and visual processing.

\section{Acknowledgements}

We would like to thank Prof. Shinsuke Hishitani who offered continuing support and constant encouragement. We would also like to thank Natsuko Henmi who made enormous contribution to conduct experiments and Dr. Gerry Quinn who gave us invaluable comments and warm encouragements.

\section{References}

Grush, R. (2004). The Emulation Theory of Representation: Motor Control, Imagery, and Perception. Behavioral and Brain Sciences, 27, 377-396. http://dx.doi.org/10.1017/S0140525X04000093

Kosslyn, S. M., Behrmann, M., \& Jeannerod, M. (1995). The Cognitive Neuroscience of Mental Imagery. Neuropsychologia, 33, 1335-1344. http://dx.doi.org/10.1016/0028-3932(95)00067-D

Logie, R. H. (1995). Visuo-Spatial Working Memory. Hove: Lawrence Erlbaum Associates Ltd. 
Pearson, G. D. (2001). Imagery and the Visuo-Spatial Sketchpad. In J. Andrade (Ed.), Working Memory in Perspective (pp. 33-59). Hove: Psychology Press Ltd.

Pearson, G. D., Logie, R. H., \& Gilhooly, K. J. (1999). Verbal Representations and Spatial Manipulation during Mental Synthesis. European Journal of Cognitive Psychology, 11, 295-314. http://dx.doi.org/10.1080/713752317

Quinn, J. G., \& Ralston, G. E. (1986). Movement and Attention in Visual Working Memory. The Quarterly Journal of Experimental Psychology Section A: Human Experimental Psychology, 38, 689-703. http://dx.doi.org/10.1080/14640748608401621

Shepard, R. N., \& Metzler, J. (1971). Mental Rotation of Three Dimensional Objects. Science, 171, 701-703. http://dx.doi.org/10.1126/science.171.3972.701

Wexler, M., Kosslyn, S. M., \& Berthoz, A. (1998). Motor Processes in Mental Rotation. Cognition, 68, 77-94. http://dx.doi.org/10.1016/S0010-0277(98)00032-8

Wohlschläger, A. (2001). Mental Object Rotation and the Planning of Hand Movements. Perception \& Psychophysics, 63, 709-718. http://dx.doi.org/10.3758/BF03194431

Wohlschläger, A., \& Wohlschläger, A. (1998). Mental and Manual Rotation. Journal of Experimental Psychology: Human Perception and Performance, 24, 397-412. http://dx.doi.org/10.1037/0096-1523.24.2.397 